\title{
Applications of Synthetic Biology in Microbial Biotechnology
}

\author{
Hal Alper, ${ }^{1}$ Patrick Cirino, ${ }^{2}$ Elke Nevoigt, ${ }^{3}$ and Ganesh Sriram ${ }^{4}$ \\ ${ }^{1}$ Department of Chemical Engineering, The University of Texas at Austin, 1 University Station, C0400, Austin, TX 78712, USA \\ ${ }^{2}$ Department of Chemical Engineering, The Pennsylvania State University, 226A Fenske Laboratory, University Park, \\ PA 16802-4400, USA \\ ${ }^{3}$ Department of Chemical and Biomolecular Engineering, University of Maryland, 1208D, \\ Chemical and Nuclear Engineering Building 090, College Park, MD 20742, USA \\ ${ }^{4}$ Molecular Biotechnology, Jacobs University gGmbH, School of Engineering and Science, Research II - Room 113, \\ Campus Ring 1, 28759 Bremen, Germany
}

Correspondence should be addressed to Hal Alper, halper@che.utexas.edu

Received 12 December 2010; Accepted 12 December 2010

Copyright () $2010 \mathrm{Hal}$ Alper et al. This is an open access article distributed under the Creative Commons Attribution License, which permits unrestricted use, distribution, and reproduction in any medium, provided the original work is properly cited.

Recent advances in synthetic biology have greatly expanded the capacity to improve pathway performance and cellular phenotype. The design, use, and importation of constructed genetic control elements, optimized genes, and functional genetic circuits can be used to modulate the function of metabolic pathways. The modularity and hierarchy of parts, devices, systems, and chassis provide a framework for studying and controlling cellular functions. Moreover, synthetic biology provides an interface where protein engineering, directed evolution, DNA synthesis, and in silico design can direct the field of metabolic engineering. Many of these advances are influencing how scientists and engineers view and perturb cellular systems. Perhaps the paramount application of these tools is in the arena of microbial biotechnology where the overarching goal is to rewire cellular systems for biochemical production. This task is quite complex given the complexity and interconnectedness of metabolism and regulation. Synthetic biology approaches provide a way to both intervene in as well as bypass cellular complexities.

This special issue contains six review articles that highlight two major aspects of synthetic biology: (1) general design principles and modeling of synthetic biology and (2) applications of synthetic biology to biochemical and biofuels production. The articles are ordered based on these two areas. In the first class, review articles describe the basic paradigm of synthetic biology and include the design and modeling of systems.
In the first article, E. Young and H. Alper discuss the areas of synthetic biology within a process engineering paradigm, through a three-tiered model that uses the central dogma of biology as a pivot. The first tier comprises the units of the central dogma and the possible flows between them (DNA, transcription, RNA, translation, and protein), the second tier includes intrinsic interactions between these units by means of regulatory mechanisms, and the third tier comprises external or environmental influences on the units. This conceptualization permits engineering approaches in synthetic biology to be classified by which components they influence. The authors provide several examples to illustrate how synthetic biology approaches can be combined toward realizing the goal of integrative synthetic biology, which the authors exemplify with two examples from the literature.

In the second article, Fritz et al. discuss a "biology by design" paradigm, which involves the conceptualization, design, and construction of a synthetic biological system, followed by the expectation that the system will perform as desired. Surmounting the challenges en route to this ambitious objective will require answering several "top-down" and "bottom-up" design questions. Bottom-up questions relate to the parts and modules of the synthetic biological system as well as their characterization and assembly in isolation, whereas top-down questions focus on desirable and undesirable interactions between the synthetic system and its biological context. This general framework can be applied to problems of increasing biological sophistication, ranging from the engineering of novel polymers, metabolic 
networks, and cellular devices. The authors illustrate the applications of these ideas to three areas: biochemical transformations, cellular devices, and therapies as well as engineering the chemistry of life.

The third paper highlights how mathematical models can predict the dynamics of a network under different conditions. This paper by Y. Zheng and G. Sriram provides detailed insights into mathematical modeling concepts and methodologies as relevant to synthetic biology. Any model is formulated on the basis of certain assumptions regarding the system under investigation as discussed by the authors. The paper introduces the two broad types of modeling frameworks: deterministic and stochastic modeling. The importance of parameter estimation and optimization in modeling is emphasized. In addition, mathematical techniques used to analyze a model such as sensitivity analysis and bifurcation analysis are presented. The authors discuss the role of modeling in phenotype analysis and conclude with three in-depth case studies about mathematical modeling in the framework of synthetic biology.

The second class of articles highlights examples and applications of synthetic biology for improved biofuels and biochemicals production. M. R. Connor and S. Atsumi review progress in improving microbial production of renewable liquid fuels and the unique role of synthetic biology in advancing these efforts. The fuel challenges facing society are first highlighted. They then provide an overview of research for the production of fermentative alcohols, nonfermentative higher alcohols, isoprenoids, and fatty acids. The importance of photosynthetic organisms in biofuels research is also discussed, and the reliance on synthetic biology tools to improve productivities and titers is emphasized.

Next, L. R. Jarboe et al. review the combined use of synthetic biology tools with traditional metabolic engineering strategies for designing and improving microbial production of chemicals from renewable resources. They begin with an overview of technologies and tools available for biocatalyst redesign. They next describe strain engineering examples in which existing pathways were modified for the overproduction of various chemicals (e.g., succinate and Dlactate). These examples are contrasted with strain re-design via the introduction of foreign or nonnatural genes and pathways for production of compounds such as L-alanine and xylitol. The latter scenario includes the use of enzymes which have been engineered to have unnatural activities.

Finally, G. H. McArthur IV and S. S. Fong focus in their paper on the impact of synthetic biology on engineering microbial metabolism. They discuss recent progress and current challenges in applying synthetic biology to metabolic engineering. In particular, the authors separately focus on each of the four workflow steps, design, modeling, synthesis, and analysis, and distinguish between parts (individual functional units) and pathways (part-based systems) for each single level.

Collectively, these papers describe the current state of synthetic biology in the context of microbial biotechnology. Current and ongoing advances in this field will certainly propel our capacity to engineer improved microorganisms in the future. In this regard, these articles all provide prospective into the future of this field.

Hal Alper

Patrick Cirino

Elke Nevoigt

Ganesh Sriram 

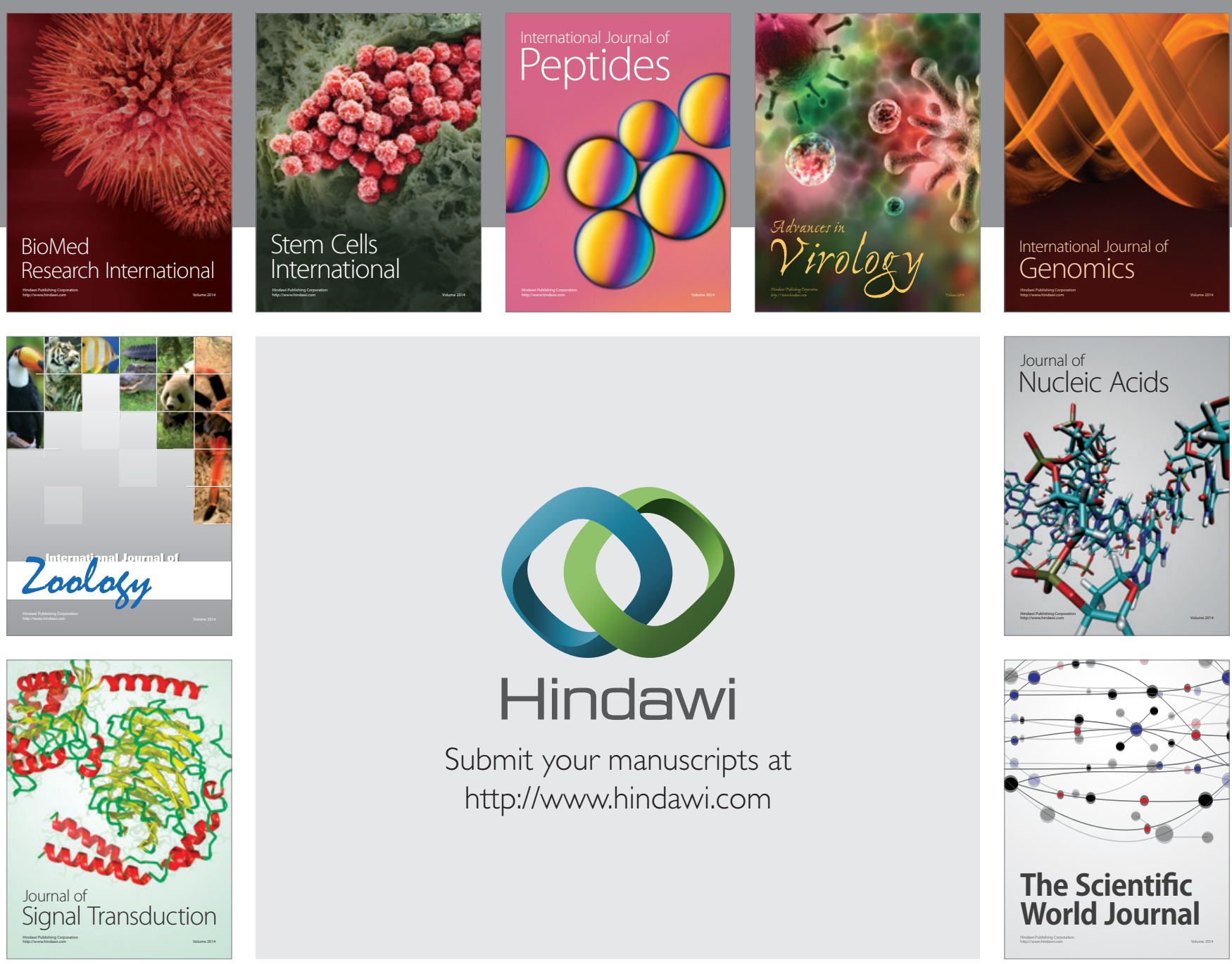

Submit your manuscripts at

http://www.hindawi.com
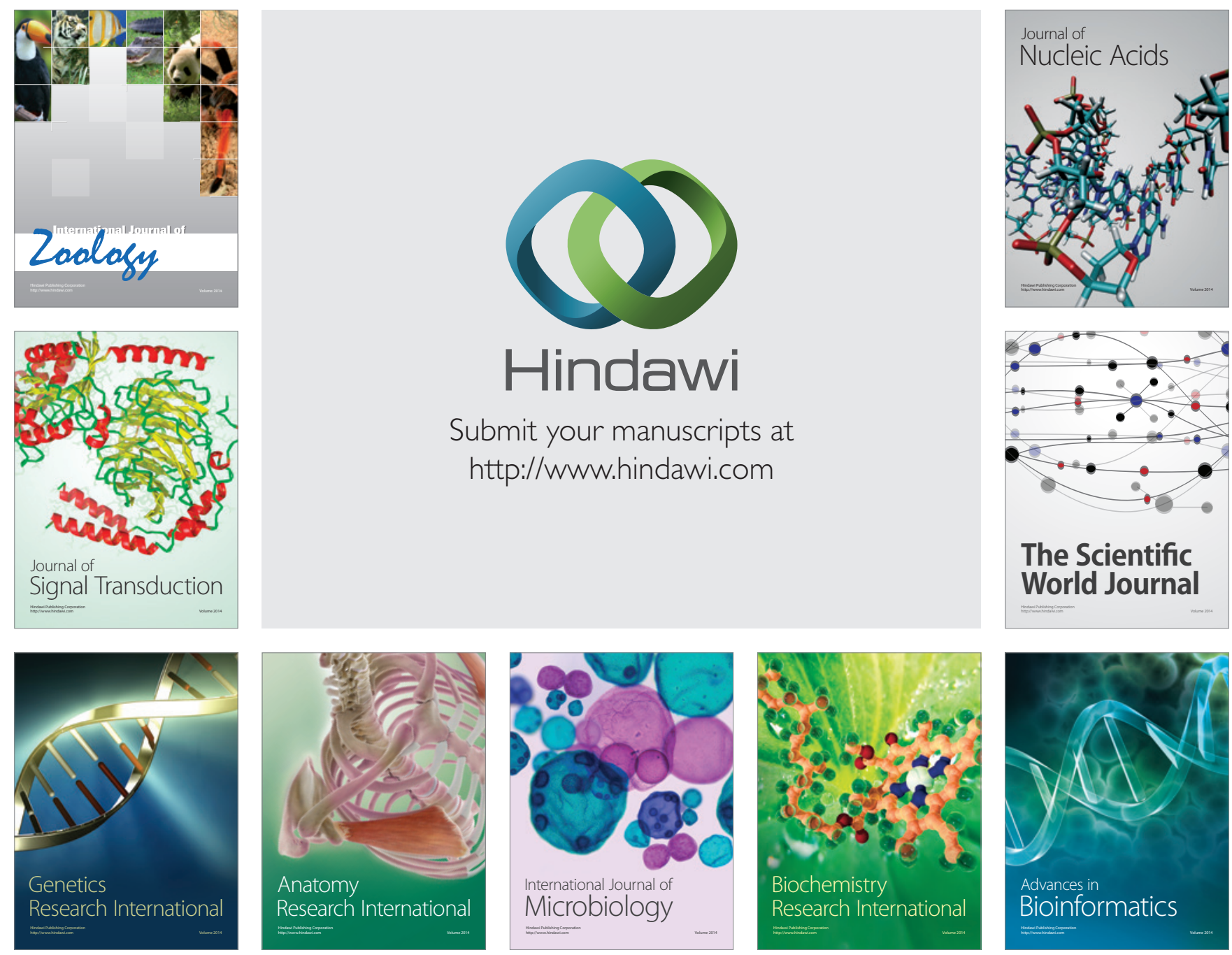

The Scientific World Journal
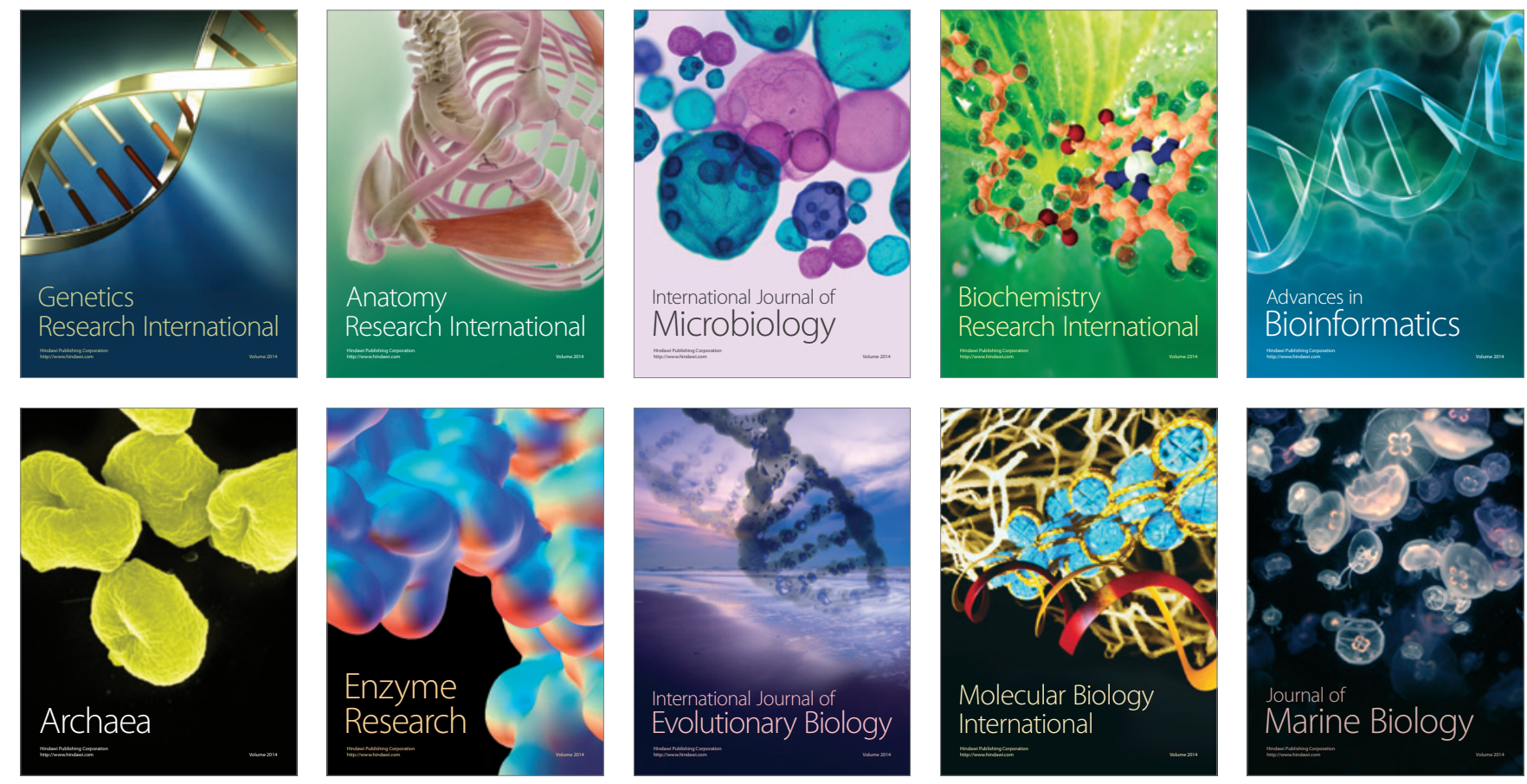